\title{
Effects of Lumbopelvic Neutralization on the Electromyographic Activity, Lumbopelvic and Knee Motion during Seated Knee Extension in Subjects with Hamstring Shortness
}

\author{
KYUE-NAM PARK, Msc, PT ${ }^{1)}$, ChUNG-HWI Yi, PhD, PT ${ }^{2)}$, \\ Hye-SEOn JeON, PhD, $\mathrm{PT}^{2)}$, Won-HweE LeE, Msc, $\mathrm{PT}^{1)}$, \\ Sung-Min Ha, Msc, PT $^{1)}$, Su-Jung KIM, Msc, PT ${ }^{1)}$, OH-Yun Kwon, PhD, PT $^{3}$ ) \\ 1) Department of Rehabilitation Therapy, Graduate School, Yonsei University \\ 2) Department of Physical Therapy, College of Health Science, Yonsei University \\ 3) Department of Physical Therapy, Kinetic Ergocise Based on Movement Analysis Laboratory, College of \\ Health Science, Yonsei University: 234 Maiji-ri, Heungup-myon, Wonju-si, Kangwon-do, 220-710 \\ Republic of Korea.TEL: +82 33-760-2721,FAX: +82 33-760-2496,E-mail: kwonoy@yonsei.ac.kr
}

\begin{abstract}
Purpose] The purpose of this study was to examine the effects of lumbopelvic neutralization on the angle of knee extension, lumbopelvic motion, and the electromyographic (EMG) activities of the quadriceps, hamstrings, and rectus abdominis muscles of subjects with hamstring shortness during seated knee extension.

[Subjects] Twenty young subjects (14 men, 6 women) with hamstring shortness were recruited for this study. [Methods] A pressure biofeedback unit was used to monitor the lumbopelvic neutralization during seated knee extension with lumbopelvic neutralization. The EMG activity and kinematic data were measured during seated knee extension with and without lumbopelvic neutralization. Surface EMG was used for measuring the muscle activities of the quadriceps, hamstrings, and rectus abdominis muscles. The angles of knee extension, lumbar flexion, lumbar rotation, and posterior pelvic tilt were measured using a 3-dimensional motion analysis system. [Results] The angles of the lumbar flexion, lumbar rotation, posterior pelvic tilt, and knee extension decreased significantly when performing seated knee extension with lumbopelvic neutralization compared to performing the same maneuver without lumbopelvic neutralization. The EMG activities of the medial and lateral hamstrings, quadriceps, and rectus abdominis decreased significantly during seated knee extension with lumbopelvic neutralization using a pressure biofeedback unit. [Conclusion] Seated knee extension exercise with lumbopelvic neutralization may be useful for minimizing compensatory lumbopelvic motion and preventing compensatory hamstrings and rectus abdominis muscle activities of subjects with hamstring shortness during seated knee extension.

Key words: Hamstring shortness, Knee extension, Lumbopelvic neutralization
\end{abstract}

(This article was submitted Jul. 12, 2011, and was accepted Aug. 27, 2011

\section{INTRODUCTION}

Knee extension exercises in the sitting position are commonly prescribed to strengthen the quadriceps, stretch shortened hamstrings, and train the control of compensatory lumbar flexion-rotation in patients with back and knee pain $^{1-5)}$. Additionally, knee extension in the sitting position is often used to classify low back pain in the movement control test ${ }^{6,7)}$. Patients with hamstring shortness can exhibit compensatory lumbopelvic motions when performing knee extension exercises due to their shortened or stiff hamstrings, and this can be prevented if the exercise is performed correctly ${ }^{2,8)}$.

Studies have emphasized the use of lumbar stabilization with an abdominal drawing-in maneuver using a pressure biofeedback unit to maintain lumbopelvic stability during hip abduction and extension performed by healthy subjects in the side-lying and prone positions ${ }^{9,10)}$. Keeping the lumbopelvic region in the neutral zone when performing lower extremity strengthening or stretching exercises and the activities of daily living has been reported to reduce the risks of cumulative tissue stress in the lumbar region, ligament injury, and posterior disc herniation ${ }^{7,11-13)}$. Keeping the neutral zone of lumbopelvic motion means that low back lordosis is similar to that of upright standing without any motions of lumbar vertebrae such as flexion, extension, rotation, or side bending ${ }^{13,14)}$.

If an individual has a lumbopelvic joint that is relatively more flexible than the hip joint, he or she will not be able to maintain the neutral zone of lumbopelvic region and lumbopelvic compensatory motion, such as lumbar flexion and/or posterior pelvic tilt, will occur during seated knee extension ${ }^{2}$. Currently, verbal instruction and tactile sensation using the hands are commonly used to monitor 
Table 1. Descriptive data of the participants, expressed as mean \pm standard deviation.

\begin{tabular}{lrrr}
\hline Variables & All $(\mathrm{n}=20)$ & Men $(\mathrm{n}=14)$ & Women $(\mathrm{n}=6)$ \\
\hline Age $(\mathrm{y})$ & $21.5 \pm 1.1$ & $22.0 \pm 0.9$ & $20.8 \pm 0.9$ \\
Body mass $(\mathrm{kg})$ & $67.7 \pm 5.7$ & $72.2 \pm 0.7$ & $61.0 \pm 0.8$ \\
Height $(\mathrm{cm})$ & $166.4 \pm 7.8$ & $172.5 \pm 1.0$ & $157.3 \pm 2.4$ \\
Seated knee extension angle $\left(^{\circ}\right)$ & $140.7 \pm 2.6$ & $140.9 \pm 3.0$ & $140.0 \pm 1.3$ \\
\hline
\end{tabular}

the lumbopelvic compensatory motions during knee extension exercises in the sitting position ${ }^{2,15,16)}$. A pressure biofeedback unit placed between the back and chair can be used to maintain lumbopelvic stability without compensatory lumbopelvic motion during seated knee extension exercise ${ }^{17}$.

While methods to prevent lumbopelvic compensatory motion during seated knee extension have been reported $^{2,17)}$, no study has investigated the effects of lumbopelvic neutralization on the electromyographic (EMG) activity of the hamstrings, quadriceps, and rectus abdominis, and the amounts of knee extension and lumbopelvic motion of subjects with short hamstrings during seated knee extension. Thus, this study compared the muscle activities of the quadriceps, hamstrings, and rectus abdominis muscles, and the angles of lumbar flexion, lumbar rotation, posterior pelvic tilt, and knee extension between seated knee extension exercise with and without lumbopelvic neutralization. We examined the effects of lumbopelvic neutralization during seated knee extension exercises in individuals with hamstring shortness, and the results should provide useful information for clinicians designing and implementing knee extension exercises for such individuals.

We hypothesized that performing seated knee extension while maintaining lumbopelvic neutralization using a pressure biofeedback unit would reduce quadriceps, hamstring and rectus abdominis muscle activities, and the amounts of knee extension and lumbopelvic motion compared with seated knee extension performed without lumbopelvic neutralization.

\section{SUBJECTS AND METHODS}

\section{Subjects}

Twenty young subjects ( 14 men, 6 women) with hamstring shortness were recruited for this study (Table 1). The presence of hamstring shortness was confirmed using the hamstring length test in the sitting position ${ }^{18)}$. Although the popliteal angle is often used to measure hamstring stiffness as a passive technique ${ }^{4)}$, Rolls and George ${ }^{18)}$ suggested that the active seated knee extension test is useful for differentiating injured subjects with hamstring injury from uninjured subjects. Therefore, we measured hamstring shortness with the active seated knee extension test. Hamstring length was measured in the seated knee extension test with the subject sitting on the edge of the therapeutic table with the hip flexed at $90^{\circ}$ and the hands placed on the lower back to prevent lumbar flexion. A goniometer was placed lateral to the knee joint and centered on the knee joint line. The stationary arm was aligned with the greater trochanter of the femur, and the moving arm with the lateral malleoli of the fibula. Keeping the foot and ankle in a relaxed position, the subject was asked to slowly extend his/her knee. The tester palpated the lower back of the subject and asked him/her to stop knee extension if the lower back was not straight. At this point the angle of knee extension was measured using the goniometer ${ }^{18}$. The mean angle of knee extension as measured with the goniometer was $140.7^{\circ}$ (Table 1). Individuals with knee and back pain were excluded from this study.

\section{Methods}

A 3-dimensional motion analysis system, (VICON MX system, Oxford Metrics, Oxford, UK) was used to measure the angles of knee extension, lumbar flexion, lumbar rotation, and posterior pelvic tilt during knee extension in the sitting position. Kinematic data sampled at $50 \mathrm{~Hz}$ were processed by Nexus 1.3 software. Reflective markers (14$\mathrm{mm}$ spheres) were placed on specific anatomical landmarks according to the guidelines of the model marker set. Five markers were placed on the lower back on the spinous processes of $\mathrm{T} 12$ and $\mathrm{L} 1$, on both the left and right sides of the spinous process of L1 and on the midpoint of the right and left posterior superior iliac spine (PSIS). Markers were placed bilaterally on the lower extremity of the anterior superior iliac spine (ASIS), lateral thigh, lateral epicondyle of the femur, lateral side of the fibula, lateral malleolus of the ankle, back of the heel, and second metatarsal bone. Lumbar flexion and rotation were calculated by constructing two segments representing the trunk and pelvis in Nexus 1.3 software. The trunk segment consisted of T12 and L1, and both the left and right sides of the spinous process of L1, while the pelvic segment consisted of the midpoint of the right and left PSIS, and both ASIS. Lumbar flexion and rotation were calculated as the angles between the trunk and pelvic segments. Posterior pelvic tilt was defined as the angle between the medio-lateral axis of the midpoint of the right and left PSIS and the sagittal plane of the pelvic segment. Kinematic data were obtained while the subject maintained isometric seated knee extension position isometrically for 5 seconds. For all kinematic variables, the mean of three measured angles obtained from 3 trials of seated knee extension with and without lumbopelvic neutralization was used for data analysis.

EMG data were collected using a wireless telemetry system (TeleMyo 2400T, Noraxon, Scottsdale, AZ, USA) and analyzed using software (MyoResearch Master Edition $1.06 \mathrm{XP}$, Noraxon). The skin at the electrode sites was prepared by shaving and then cleaning with alcohol. Surface electrode pairs were positioned at an interelectrode distance of $2 \mathrm{~cm}$, and the reference electrode was placed on 
the patella. EMG data were collected for the following muscles: vastus medialis oblique $(2 \mathrm{~cm}$ medially from the superior rim of the patella), vastus lateralis (lateral surface of the lower third of the thigh, at approximately a $20^{\circ}$ angle from vertical), rectus femoris (medial anterior surface of the thigh, approximately halfway between the hip and the knee), medial hamstring ( $3 \mathrm{~cm}$ in from the lateral border of the thigh and approximately halfway from the gluteal fold to the back of the knee), lateral hamstring ( $2 \mathrm{~cm}$ apart parallel to the muscle fibers on the lateral aspect of the thigh at two-thirds the distance between the trochanter and the back of the knee), and rectus abdominis (approximately $2 \mathrm{~cm}$ lateral and across from the umbilicus over the muscle belly $)^{19)}$. A wood board $(45 \mathrm{~cm} \times 38 \mathrm{~cm})$ with hole $(18 \mathrm{~cm}$ $\times 12 \mathrm{~cm}$ ) was placed on the chair for attaching electrodes to the hamstrings.

The sampling rate was $1000 \mathrm{~Hz}$. The raw signal was fullwave rectified and filtered between $20 \mathrm{~Hz}$ and $500 \mathrm{~Hz}$ using a bandpass digital filter (Lancosh FIR). The root-meansquare values of the raw data were calculated, with the mean RMS value of three trials relative to the reference voluntary contraction (RVC) calculated for each muscle. According to a previous study, the RVC method requires less time and effort for subject to perform the task for RVC and can effectively eliminate the effects of factors such as electrode placement or skin conduction, compared to the traditional maximal voluntary isometric contraction (MVIC) method which requires that subjects perform muscle contraction maximally for each muscle and trial ${ }^{20)}$.

The RVC was calculated for the quadriceps with the subject performing full knee extension with a $3-\mathrm{kg}$ sandbag placed on the ankles in the sitting position, for the hamstring with the subject performing $30^{\circ}$ of knee flexion with a $3-\mathrm{kg}$ sandbag placed on the ankles in the prone position, and for the rectus abdominis with the subject performing a curl-up up to the inferior angle of both scapula in the hook-lying position. For each RVC, the EMG signal was collected for 3 seconds whilst maintaining each position. The data for each trial were expressed as percentages of the calculated mean RMS values of the RVC (\%RVC), and the mean \%RVC of three trials was used for analysis.

Prior to the experiments, the principal investigator explained all of the procedures to the subjects in detail. All subjects signed an informed consent form approved by the Institute Review Board of Yonsei University. Before testing, the subjects were taught how to adopt a neutral lumbopelvic position while sitting and practiced it. Lumbopelvic neutralization was defined as the position of lumbar spine without visible lumbar flexion, extension, rotation or side bending of any of the lumbar vertebrae ${ }^{21)}$. The subject assumed a sitting position on a chair with the upper trunk and lumbopelvic region aligned along a straight line. The hip joint was maintained at $90^{\circ}$ during the knee extension exercise. The subjects were familiarized with the use of a pressure biofeedback unit (Chattanooga Group, Hixson, TN) for lumbopelvic neutralization. Each subject practiced lumbopelvic neutralization using the pressure biofeedback unit and was taught about the pressure-monitoring mechanism of the pressure biofeedback unit. The training session lasted approximately 15 minutes. The EMG activity and kinematic data (angles of knee extension, lumbar flexion, lumbar rotation, and posterior pelvic tilt) were measured during seated knee extension with and without lumbopelvic neutralization. Data were collected at the endrange movement of seated knee extension for 5 seconds. The test order was selected randomly. A 10-minute rest period was allowed to minimize muscle fatigue and the testing effect between tests.

In experiments without lumbopelvic neutralization, the inelastic bag of the pressure biofeedback unit was inflated to $70 \mathrm{mmHg}$ and placed between the back of the chair and the subject's lower back. The subject was instructed to not monitor the pressure gauge. The subject was asked to perform maximum knee extension in the sitting position while the thigh and knee joints remained in contact with the chair to prevent hip flexion.

The protocol for the experiments with lumbopelvic neutralization were the same as that without lumbopelvic neutralization, except that the subject was asked to maintain a pressure of $70 \mathrm{mmHg}$ using visual feedback from an analog pressure gauge during seated knee extension. The data collected when pressure changes were less than 2 $\mathrm{mmHg}$ during seated knee extension were used for the statistical analysis. Because minimal change of the pressure (less than $2 \mathrm{mmHg}$ ) was observed during respiration at rest ${ }^{22}$, EMG activity and kinematic data were measured for 5 seconds during seated knee extension with lumbopelvic neutralization.

The sample size needed to ensure protection from type II error was calculated. Among the variables, the one with the largest standard deviation during pilot studies was chosen for the power analysis. A power of $80 \%$ and an $\alpha$ level of 0.05 were assumed and the difference of the means between the two conditions (i.e., with versus without lumbopelvic neutralization) needed to be at least $10 \%$ of RVC and $5^{\circ}$, which required that at least 15 subjects were tested. All measurement variables are expressed as the mean and standard deviation (SD). The significance of differences between the two conditions was assessed using the paired t-test and the level of statistical significance was chosen as 0.05 . Pearson's correlation coefficient was calculated to identify significant relationships between the knee extension angle and the lumbar flexion and rotation angles.

\section{RESULTS}

The mean difference was $38.17^{\circ}$ for knee extension $(95 \%$ CI 36.96 to $\left.39.37^{\circ}, \mathrm{p}<0.01\right), 24.33^{\circ}$ for lumbar flexion $(95 \%$ CI 22.40 to $\left.26.27^{\circ}, \mathrm{p}<0.01\right), 10.97^{\circ}$ for lumbar rotation (95\% CI 10.23 to $11.71^{\circ}, \mathrm{p}<0.01$ ), and $10.13^{\circ}$ for posterior pelvic tilt $\left(95 \%\right.$ CI 8.71 to $\left.11.55^{\circ}, \mathrm{p}<0.01\right)$; the decrease was significant when performing seated knee extension with lumbopelvic neutralization (Table 2).

In seated knee extension with lumbopelvic neutralization, there were significant reductions in the EMG activities, expressed as the \%RVC, of the vastus medialis obliquus by 71.76 (95\% CI 63.54 to $79.98, \mathrm{p}<0.01)$, vastus lateralis by $64.88(95 \% \mathrm{CI} 56.44$ to $73.31, \mathrm{p}<0.01)$, rectus femoris by $50.96(95 \% \mathrm{CI} 41.60$ to $60.31, \mathrm{p}=0.002)$, medial 
Table 2. Comparison of kinematic data between with and without lumbopelvic neutralization

\begin{tabular}{lccc}
\hline & \multicolumn{2}{c}{ Angle $\left(^{\circ}\right)$ Mean \pm SD } & \\
\cline { 2 - 3 } Movements & $\begin{array}{c}\text { Without } \\
\text { lumbopelvic neutralization }\end{array}$ & $\begin{array}{c}\text { With } \\
\text { lumbopelvic neutralization }\end{array}$ & $\begin{array}{c}\text { Mean Difference } \\
(95 \% \text { CI })\end{array}$ \\
\hline Lumbar flexion & $27.6 \pm 4.7^{*}$ & $3.3 \pm 0.6^{*}$ & $24.3(22.4-26.3)$ \\
Lumbar rotation & $11.4 \pm 4.7^{*}$ & $0.5 \pm 0.2^{*}$ & $11.0(10.2-11.7)$ \\
Posterior pelvic tilt & $10.2 \pm 3.0^{*}$ & $0.1 \pm 0.1^{*}$ & $10.1(8.7-11.6)$ \\
Knee extension & $179.2 \pm 0.8^{*}$ & $141.0 \pm 2.4^{*}$ & $38.2(37.0-39.4)$ \\
\hline
\end{tabular}

NOTE. Mean differences were calculated by subtracting the lumbopelvic neutralization value from the without lumbopelvic neutralization value. Abbreviations: $\mathrm{CI}$; confidence interval. ${ }^{*} \mathrm{p}<0.01$.

Table 3. Comparison of EMG amplitude between with and without lumbopelvic neutralization

\begin{tabular}{lccc}
\hline \multirow{2}{*}{ Muscles } & \multicolumn{2}{c}{$\%$ RVC } & Mean $\pm \mathrm{SD}$ \\
\cline { 2 - 3 } & $\begin{array}{c}\text { Without } \\
\text { lumbopelvic neutralization }\end{array}$ & $\begin{array}{c}\text { With } \\
\text { lumbopelvic neutralization }\end{array}$ & $\begin{array}{c}\text { Mean difference } \\
(95 \% \mathrm{CI})\end{array}$ \\
\hline VMO & $89.3 \pm 14.1^{*}$ & $17.5 \pm 5.1^{*}$ & $71.8(63.6-80.0)$ \\
Vastus lateralis & $82.5 \pm 16.8^{*}$ & $17.6 \pm 3.8^{*}$ & $64.9(56.4-73.3)$ \\
Rectus femoris & $76.5 \pm 21.3^{*}$ & $25.5 \pm 8.1^{*}$ & $51.0(41.6-60.3)$ \\
Medial hamstring & $15.0 \pm 4.4^{*}$ & $5.0 \pm 2.7^{*}$ & $10.1(8.0-12.1)$ \\
Lateral Hamstring & $38.4 \pm 12.4^{*}$ & $16.7 \pm 2.9^{*}$ & $26.4(21.0-31.8)$ \\
Rectus abdominis & $16.7 \pm 2.9^{*}$ & $4.1 \pm 1.9^{*}$ & $12.6(11.0-14.3)$ \\
\hline
\end{tabular}

NOTE. Mean differences were calculated by subtracting the lumbopelvic neutralization value from the without lumbopelvic neutralization value. Abbreviations: $\mathrm{CI}$; confidence interval, VMO; vastus medialis oblique. ${ }^{*} \mathrm{p}<0.01$.

hamstring by $10.05(95 \% \mathrm{CI} 7.96$ to $12.14, \mathrm{p}<0.01)$, lateral hamstring by $26.40(95 \% \mathrm{CI} 21.01$ to $31.80, \mathrm{p}=0.001)$, and rectus abdominis by 12.62 (95\% CI 10.98 to $14.26, \mathrm{p}<0.01)$ (Table 3).

There were a significant negative Pearson's correlations between the length of the hamstrings and the angle of lumbar flexion and lumbar rotation measured during seated knee extension without lumbopelvic neutralization $(\mathrm{r}=$ $-0.64, p<0.01$ and $r=-0.48, p<0.05$, respectively).

\section{DISCUSSION}

The main finding of this study was that the angles of lumbar flexion, lumbar rotation, and posterior pelvic tilt during seated knee extension were significantly higher without lumbopelvic neutralization than with lumbopelvic neutralization. According to Sahrmann, when the quadriceps contract to extend the knee joint in the sitting position, the lumbar spine flexes and the pelvis tilts posteriorly in subjects with hamstring stiffness and shortness ${ }^{2}$. If an individual has greater flexibility in the lumbopelvic region than in the hamstrings, compensatory motion will occur at the relatively flexible lumbopelvic joint while performing forward bending in the standing position, straight leg raising in the supine position or knee extension in the sitting position. We found significant negative correlations between the length of the hamstrings and the angles of lumbar flexion and rotation during seated knee extension, which supports our hypothesis that hamstring shortness contributes to compensatory lumbopelvic motion when performing knee extension in the sitting position.

Performing knee extension in the presence of hamstring shortness may increase the load on the lumbar spine. In our study, the angles of lumbar flexion, rotation, and posterior pelvic tilt increased significantly during seated knee extension without lumbopelvic neutralization. Repeatedly performing knee extension or activities without lumbopelvic neutralization may result in excessive loading of the lumbar spine, eventually leading to cumulative lowback injury. Lumbopelvic neutralization with a pressure biofeedback unit can be used to minimize the compensatory lumbopelvic motion when subjects with hamstring shortness are performing knee extension. Thus, when individuals with hamstring shortness perform seated knee extension to stretch the hamstrings or strengthen the quadriceps, lumbopelvic neutralization should be considered to reduce excessive loading on the lumbar spine and allow the exercise to be performed correctly.

The angle of knee extension during the seated knee extension test was $38^{\circ}$ lower in the presence of lumbopelvic neutralization than without lumbopelvic neutralization. The hamstrings are attached to the ischial tuberosity and tilt the pelvis posteriorly when the knee joint is fixed ${ }^{23)}$. The decreased angle of posterior pelvic tilt with lumbopelvic neutralization may contribute to increased hamstring stiffness, which may limit the angle of knee extension. Another reason for the reduced angle of knee extension is the influence of passive insufficiency when extending the knee joint at the end-range of seated knee extension in the presence of lumbopelvic neutralization.

Decreasing the angle of posterior pelvic tilt during seated knee extension with lumbopelvic neutralization may be effective for selective stretching and muscle length testing of the hamstrings. Stabilization of the proximal part of the shortened muscle is essential for stretching the shortened muscle and accurately determining the muscle length ${ }^{24,25)}$. 
Effective stabilization with lumbopelvic neutralization may effectively change the length of the hamstring during seated knee extension. In the absence of lumbopelvic neutralization, the muscle fibers of the hamstring may not be stretched effectively due to insufficient fixation of the proximal part of the hamstring during seated knee extension.

In this study, the muscle activities of the quadriceps, hamstrings, and rectus abdominis during seated knee extension were significantly lower with lumbopelvic neutralization than without lumbopelvic neutralization. Decreased quadriceps muscle activity can be explained by the decrease in the length of the knee extension moment arm, due to the decreased angle of knee extension during seated knee extension with lumbopelvic neutralization. This decreased moment arm may contribute to the reduction in activity of the quadriceps in the presence of lumbopelvic neutralization ${ }^{26)}$. Discomfort in the hamstrings may increase the difficulty of contracting the hamstrings and quadriceps ${ }^{27}$. A pulling sensation or discomfort may influence muscle activity ${ }^{28)}$. In the last phase of seated knee extension, greater discomfort from the pulling sensation of a stretched hamstring with lumbopelvic neutralization than without lumbopelvic neutralization may inhibit additional quadriceps and hamstring muscle activation.

The hamstrings act to tilt the pelvis posteriorly when the knee joint is fixed ${ }^{23)}$. Decreased posterior pelvic tilt in the presence of lumbopelvic neutralization may reduce the activity of the hamstrings, which is another reason why hamstring muscle activity is lower in the presence of lumbopelvic neutralization than without lumbopelvic neutralization.

Contraction of the rectus abdominis muscle induces lumbar flexion during hip flexion or knee extension in the sitting position ${ }^{2,29)}$. Reduced lumbar flexion in the presence of lumbopelvic neutralization may decrease the rectus abdominis muscle activity.

Our study confirms the hypothesis that lumbopelvic neutralization using a pressure biofeedback unit has the favorable effects of preventing compensatory lumbar flexion, lumbar rotation, posterior pelvic tilt, and unnecessary activity in muscles, such as the hamstrings and rectus abdominis, during seated knee extension in individuals with hamstring shortness. However, this study had several limitations. First, the results from our young population might not be generalizable to other populations. Second, we could not measure the activities of the erector spinae, external oblique, internal oblique, or transverse abdominis muscles, which are lumbar stabilizers. Thus, future studies should investigate the effects of lumbopelvic neutralization using a pressure biofeedback unit on the deep and superficial back extensor and abdominal muscle activities during seated knee extension in subjects with hamstring shortness. Additionally, the effects of lumbopelvic neutralization using a pressure biofeedback unit during seated knee extension on lengthening the hamstrings and strengthening the quadriceps should be studied in subjects with hamstring shortness.

\section{REFERENCES}

1) Marques AP, Vasconcelos AA, Cabral CM, et al.: Effect of frequency of static stretching on flexibility, hamstring tightness and electromyographic activity. Braz J Med Biol Res, 2009, 42: 949-953.

2) Sahrmann SA: Diagnosis and Treatment of Movement Impairment Syndrome. 1st ed. Missouri: Mosby, 2002,pp 103-111.

3) Tagesson S, Oberg B, Good L, et al.: A comprehensive rehabilitation program with quadriceps strengthening in closed versus open kinetic chain exercise in patients with anterior cruciate ligament deficiency: A randomized clinical trial evaluating dynamic tibial translation and muscle function. Am J Sports Med, 2008, 36: 298-307.

4) White LC, Dolphin P, Dixon J: Hamstring length in patellofemoral pain syndrome. Physiotherapy, 2009, 95: 24-28.

5) Williams GN, Snyder-Mackler L, Barrance PJ, et al.: Quadriceps femoris muscle morphology and function after ACL injury: A differential response in copers versus non-copers. Biomech, 2005, 38: 685-693.

6) Luomajoki H, Kool J, de Bruin ED, et al.: Movement control tests of the low back; Evaluation of the difference between patients with low back pain and healthy controls. BMC Musculoskelet Disord, 2008, 24: 170-181.

7) Maluf KS, Sahrmann SA, Van Dillen LR: Use of a classification system to guide nonsurgical management of a patient with chronic low back pain. Phys Ther, 2000, 80: 1097-1111.

8) Van Dillen LR, Sahrmann SA, Wagner JM: Classification, intervention, and outcomes for a person with lumbar rotation with flexion syndrome. Phys Ther, 2005, 85: 336-351.

9) Cynn HS, Oh JS, Kwon OY, et al.: Effects of lumbar stabilization using a pressure biofeedback unit on muscle activity and lateral pelvic tilt during hip abduction in sidelying. Arch Phys Med Rehabil, 2006, 87: 1454-1458.

10) Oh JS, Cynn HS, Won JH, Kwon OY, et al.: Effects of performing an abdominal drawing-in maneuver during prone hip extension exercises on hip and back extensor muscle activity and amount of anterior pelvic tilt. J Orthop Sports Phys Ther, 2007, 37: 320-324.

11) Dolan P, Adams MD: Effects of lumbar and hip mobility on the bending stresses acting on the lumbar spine. Clin Biomech, 1993, 8: 185-192.

12) McGill SM, Kippers V: Transfer of loads between lumbar tissues during the flexion-relaxation phenomenon. Spine, 1994, 19: 2190-2196.

13) Suni J, Rinne M, Natri A, et al.: Control of the lumbar neutral zone decreases low back pain and improves self-evaluated work ability: A 12-month randomized controlled study. Spine, 2006, 31: E611-E620.

14) Harris-Hayes M, Van Dillen LR, Sahrmann SA: Classification, treatment and outcomes of a patient with lumbar extension syndrome. Physiother Theory Pract, 2005, 21: 181-196.

15) O'Sullivan PB, Dankaerts W, Burnett AF, et al.: Effect of different upright sitting postures on spinal-pelvic curvature and trunk muscle activation in a pain-free population. Spine, 2006, 31: 707-712.

16) O'Sullivan PB, Grahamslaw KM, Kendell M, et al.: The effect of different standing and sitting postures on trunk muscle activity in a pain-free population. Spine, 2002, 27: 1238-1244.

17) Richardson CA, Hodges PW, Hides JA: Therapeutic exercise for lumbopelvic stabilization: a motor control approach for the treatment and prevention of lowback pain. 2nd ed. Edinburgh: Churchill Livingstone, 2004, pp 153-154.

18) Rolls A, George K: The relationship between hamstring muscle injuries and hamstring muscle length in young elite footballers. Phys Ther Sport, 2004, 5: $179-187$.

19) Cram JR, Kasman GS, Holtz J: Introduction to Surface Electromyography. 1st ed. Maryland: Aspen Publishers Inc, 1998, pp 365-370.

20) Hsu YH, Chen WY, Lin HC, et al.: The effects of taping on scapular kinematics and muscle performance in baseball players with shoulder 
impingement syndrome. J Electromyogr Kinesiol, 2009, 19:1092-1099.

21) Van Dillen LR, Sahrmann SA, Norton BJ, et al.: Reliability of physical examination items used for classification of patients with low back pain Phys Ther ,1998, 78: 979-988.

22) Roussel N, Nijs J, Truijen S, et al.: Altered breathing patterns during lumbopelvic motor control tests in chronic low back pain: a case-control study. Eur Spine J, 2009,18:1066-1073.

23) Neumann DA: Kinesiology of the musculoskeletal system. 1st ed. Philadelphia: Mosby, 2002,pp 418-420.

24) Kendall FP, McCreary EK, Provance PG: Muscles: Testing and function with posture and pain. 5th ed. Maryland: Williams \& Wilkins, 2005, pp 39 41.
25) Kisner C, Colby LA: Therapeutic exercise: foundations and techniques. 4th ed. Pennsylvania: F.A. Davis, 2002, pp 171-175.

26) Krishnan C, Williams GN: Sex differences in quadriceps and hamstrings EMG-moment relationships. Med Sci Sports Exerc, 2009, 41: 1652-1660.

27) Keir PJ, Wells RP, Ranney DA: Passive properties of the forearm musculature with reference to hand and finger postures. Clin Biomech, 1996, 11: 401-409.

28) Cesarelli M, Bifulco P, Bracale M: Quadriceps muscles activation in anterior knee pain during isokinetic exercise. Med Eng Phys, 1999, 21: 469-478.

29) Van Dillen LR, Sahrmann SA, Norton BJ: Movement system impairmentbased categories for low back pain: stage 1 validation. J Orthop Sports Phys Ther, 2003, 33: 126-142. 